\title{
Biomimetic Toothpastes: Remineralization Potential of Deciduous Teeth ${ }^{+}$
}

\author{
Flavia Iaculli 1, *, Alessandro Salucci ${ }^{~}$, Chiara Silvestri ${ }^{1}$, Gaetano Ierardo ${ }^{1}$, Roberto Matassa ${ }^{2}$, \\ Antonella Polimeni ${ }^{1}$, Maurizio Bossù ${ }^{1}$ \\ 1 Department of Oral and Maxillofacial Science, Sapienza University of Rome, 00185 Rome, Italy; \\ alessandro.salucci@uniroma1.it (A.S.); chiarasilvestri92@gmail.com (C.S.); gaetano.ierardo@uniroma1.it \\ (G.I.); antonella.polimeni@uniroma1.it (A.P.); maurizio.bossu@uniroma1.it (M.B.) \\ 2 Section of Human Anatomy, Department of Anatomical, Histological, Forensic and Orthopaedic Sciences, \\ Sapienza University of Rome, 00185 Rome, Italy; roberto.matassa@uniroma1.it \\ * Correspondence: flavia.iaculli@uniroma1.it \\ † Presented at 1st International Electronic Conference on Applied Sciences, 10-30 November 2020; Available \\ online: https://asec2020.sciforum.net/.
}

Published: 10 November 2020

\section{Oral Presentation:}

Human teeth are constantly subjected to a continuous process of de- and remineralization, mostly due to Ph alteration, cariogenic bacteria, external agent, heat, cold, sweet as well as erosive food and drink.

The main issue concerning demineralization is that loss of mineralized dental tissues favors the development of demineralized area that may evolve in dental decay.

This aspect is mainly true for primary teeth, since the enamel of deciduous teeth appears to be thinner than the permanent ones, lesser mineralized (mineralization of $80.6 \%$ in deciduous enamel and $89.7 \%$ in permanent enameled teeth) with not completely formed prisms at the outer layer. As you can see in the right side of my screen, the percentages of mineralization are slightly different between deciduous and permanent enamel.

So, in order to prevent teeth demineralization and promote re-mineralization, several biomaterials have been used with the aim to restore enamel and dentine tissues.

Particularly, the ability of fluorine to re-mineralize dental surfaces has been widely demonstrated within the scientific literature for both permanent and deciduous teeth, and, among the employed biomaterials commercially available, toothpastes are the mainly used agents in preventive dentistry.

Fluoride-containing toothpastes, have been successfully introduced as a global solution to mostly prevent dental caries and promote tooth re-mineralization.

However, the potential risk of fluorosis due to high intake should be taken into account and a great attention has to be paid to fluoride toothpaste concentrations as well as systemic supply.

Dental fluorosis occurs as a result of excess fluoride ingestion during tooth formation and mainly involved the permanent teeth.

The risk of fluorosis is mostly real in children of less than 5 years of age, due to the swallowing of a high amount of fluoride toothpaste during the mineralization phase of permanent teeth. To balance the fluorine intake, you can see an example of toothpaste administration to prevent fluorosis, according to the national guidelines of Italian health service, in respect to the age of the little patients.

Thus, to minimize the risk of fluorosis occurrence, newly developed biomimetic toothpastes have been proposed as valid alternative, providing promising results in preventive dentistry.

Therefore, in light of these consideration, the aim of the present study was to analyze the ability of commercial biomimetic toothpastes to diffuse into deciduous enamel layer to remineralize the crystal habitat. These aspects not only would improve the synthesis of novel biomaterials specifically 
designed for deciduous teeth, but also would represent a positive global economic impact because of the wide prevalence of dental caries affecting primary teeth.

To evaluate the ability of toothpastes to diffuse into the deciduous enamel layer, 8 primary teeth were extracted as a result of orthodontic treatments or physiological replacements. Once extracted, each element was preserved in normal saline solution and then divided at the cementum-enamel junction level, by means of diamond-tipped saw Secotron 200, in order to remove the root portion. Subsequently, the specimens were fixed on composite resin supports and subjected to etching with $37 \%$ orthophosphoric acid for $1 \mathrm{~min}$ to reproduce the physiological demineralization that occurs in the oral environment, and then rinsed with normal saline solution.

Two teeth per group were manually brushed for 15 days, three times a day, using 3 different pediatric toothbrushes (one for each group) for $2 \mathrm{~min}$, as follows: (a) commercial toothpaste containing fluorine $500 \mathrm{ppm}$; (b) commercial toothpaste containing fluorine $1400 \mathrm{ppm}$; (c) toothpaste containing hydroxyapatite nanocrystal. Two elements used as control were subjected to the same manual brushing with water, 3 times a day for 15 days. After every treatment, the samples were rinsed and preserved at room temperature in normal saline solution, which was renewed every brushing session.

Then, specimens were directly settled onto a carbon planchet stub without conductive coating and the obtained cross-sections were not flattened and polished. A thin film of distilled water was deposited on the hydrated samples at room temperature. Thus, All samples were observed by means of variable pressure scanning electron microscopy (VP-SEM) and energy dispersive X-ray spectroscopy (EDS).

Cross-sectioned surfaces of deciduous teeth showed well-formed and aligned microrods interspaced by an enamel matrix

From a morphological point-of-view, cross-sectioned surfaces of deciduous teeth showed wellformed and aligned microrods interspaced by an enamel matrix.

The corresponding EDS spectra demonstrated the following $\mathrm{Ca} / \mathrm{P}$ ratio $(1.84 \pm 0.51 \%$ after $\mathrm{HN}$ treatment, $1.75 \pm 0.65 \%$ after F1400 treatment and $1.65 \pm 0.45 \%$ after F500 treatment).

Evaluating the chemical spatial distribution across the enamel layer, the EDS analysis confirmed the absorption of the nanostructured $\mathrm{HN}$ material through the outer enamel surface until a penetration depth of about $16.10 \pm 0.75$ and $15.64 \pm 0.68 \mu \mathrm{m}$ of $\mathrm{P}$ and $\mathrm{Ca}$, respectively.

On the other hand, both F1400 and F500 were able to diffuse only microparticles of silicate through the enamel layer with a penetration depth slightly higher in case of F500 toothpaste. However, statistically significant differences were not observed among groups.

According to the obtained results, it seemed that after hydroxyapatite toothpaste brushing, the increasing calcium phosphate deposits qualitatively confirm the occurred penetration of the toothpaste within the outermost enamel layer and would led to a growing of the crystal habitat into deciduous enamel, as a biomimetic crystallization effect. On the contrary, F1400 and F500 biomaterials provide a relative stable $\mathrm{Ca} / \mathrm{P}$ ratio through dental enamel due to the lack of calcium and phosphate contents within the toothpastes.

So it can be concluded that, since the chemical and dimensional constituents of toothpaste containing hydroxyapatite nanocrystal have shown to better diffuse in the enamel layer, these type of material may potentially contribute to remineralize the early stage or the loss of the enamel in deciduous teeth. On the other hand, phosphate and calcium were not detected in F1400 and F500, however, the high amount of silicate might confer some protective action decreasing the porosity and structural weakness of tooth.

In addition, it should be stressed that the abrasive potential of fluoride toothpastes due to the presence of silicate microaggregate is underestimated and should be further studied in depth. Instead, low abrasion may be obtained with toothpaste containing hydroxyapatite nanocrystal thanks to the softness of chemical aggregates of calcium carbonate.

The preliminary results obtained by the present study, suggested a promising remineralization of toothpastes containing nanocrystals of hydroxyapatite. This aspect should be studied in depth, not only to further support the supplied outcomes, but also to provide a reliable alternative to toothpaste 
containing fluorine that, although having valuable protective action, may cause fluorosis as a result of excessive assumption.

These findings will be useful in the current research to tailor dental materials with specific properties and to develop controlled toothpaste able to possess protective and biomineralization features and to be used in different applications of tissue regeneration and preventive dentistry.

Keywords: Biomimetic toothpastes; Dental caries; Enamel; Preventive dentistry; Primary teeth; Tooth remineralization

Publisher's Note: MDPI stays neutral with regard to jurisdictional claims in published maps and institutional affiliations.

(C) 2020 by the authors. Submitted for possible open access publication under the terms and conditions of the Creative Commons Attribution (CC BY) license

(http://creativecommons.org/licenses/by/4.0/). 